\title{
Impact de la perturbation par les interventions sylvicoles et le feu sur la dynamique d'un peuplement forestier en zone semi-décidue de Côte d'Ivoire
}

\author{
Memel S. C. Yedmel ${ }^{1,2^{*}}$, Yao Sadaiou S. Barima ${ }^{2}$, N'Guessan F. Kouamé ${ }^{1}$ \& Nicolas BarbieR ${ }^{3}$ \\ 'Université de Cocody-Abidjan (Côte d'Ivoire) ; Laboratoire de Botanique, UFR Biosciences. 22 BP 582 Abidjan 22, Côte d'Ivoire. \\ ${ }^{2}$ Université Libre de Bruxelles (Belgique); Service Evolution Biologique et Ecologie. CP160/12, avenue F.D. Roosevelt 50, 1050 Bruxelles. \\ ${ }^{3}$ Université Libre de Bruxelles (Belgique), Laboratoire de Complexité et Dynamique des Systèmes Tropicaux. - Campus du \\ Solbosch, CP 169, Avenue F.D. Roosevelt 50, B-1050 Bruxelles. \\ *Auteur pour les correspondances (Email : yscharles@yahoo.fr et myedmel@ulb.ac.be) \\ Reçu le 07-05-2009, accepté le17-06-2010.
}

\begin{abstract}
Résumé
Le dispositif permanent de la Téné (Côte d'Ivoire) est un peuplement forestier aménagé depuis 1978 afin d'étudier, à long terme, la dynamique des essences à potentiel commercial. En 1983, le dispositif a été parcouru à plus de $65 \%$ par un feu de forêt. L'objectif de la présente étude est de décrire l'effet de la perturbation occasionnée par les interventions sylvicoles et le passage du feu sur la dynamique du peuplement.

Les résultats obtenus et les tests statistiques appliqués ont montré une homogénéité entre les parcelles avant les modalités sylvicoles tandis qu'après celle-ci une différence significative est observée entre les parcelles témoins et traitées. L'évolution de la surface terrière, au cours du temps, montre deux grandes périodes dont la première située entre 1978 à 1982 correspond à l'effet traitement et la seconde traduit par l'effet du feu après 1986. L'impact de la perturbation s'est fait aussi sentir en 1978 sur la surface terrière des essences commerciales tandis qu'au delà de 1994, cet effet s'est fait au profit des essences secondaires. Ces résultats montrent que les interventions sylvicoles supposées favoriser l'accroissement, en volume, des essences commerciales peuvent être vulnérables au feu et entraîner à long terme des effets majeurs sur la dynamique du peuplement.
\end{abstract}

Mots clés : exploitation forestière, éclaircie, feu de forêt, traitements sylvicoles, composition floristique.

\section{Abstract}

Impact of the disturbance by the silvicultural treatments and fire on the dynamics of semi-deciduous forest of Côte d'lvoire

The Téné permanent plots (Côte d'Ivoire) are forest research devices set up in 1978, to study on the long term the dynamics of the timber species. In 1983, the plots were accidentally burned and more than $65 \%$ of the total areas were affected. The aim of this study is to describe effects of the disturbance caused by the thinning and the fire on the dynamics of the forest.

The results obtained and the statistical tests applied showed homogeneity between plots before the thinning while after this a significant difference is observed between the treated plots and the control plots. The basal area shows two great periods whose the first located between 1978 to 1982 corresponds to the treatment effect and the second is expressed by the effect of fire after 1986. The impact of the disturbance is also felt in 1978 on the basal area of the timber species while since 1994 there is a profit of the non timber species. These results show that the silvicultural methods supposed to the increase volume of the timber species can be vulnerable to fire and influence in the long time majors effects on forest dynamics.

Keywords: logging, thinning, fire of forest, silvicultural treatments, floristic composition. 


\section{Introduction}

L'avenir des forêts tropicales est devenu un sujet de préoccupation majeur (Poore et al., 1989). L'accord signé en 1900 (objectif 2000) par les 58 membres de l'Organisation Internationale des Bois Tropicaux (OIBT), stipulait qu'en l'an 2000, la totalité des bois tropicaux commercialisés dans le monde devait provenir des forêts aménagées de manière durable. En 1992 au sommet de la Terre à Rio, la première déclaration internationale sur les forêts insistait sur la nécessité de préserver les multiples services fournis par les écosystèmes forestiers, en renforçant leur protection, leur gestion et leur conservation. Concilier la préservation et l'exploitation des forêts tropicales, pour à la fois préserver leur rôle écologique et satisfaire les besoins des pays et des populations locales, constitue un véritable défi (Flores, 2005). Les surfaces de forêts consacrées à la préservation sont encore considérées comme insuffisantes et connaissent en plus, des difficultés de gestion.

Pearce et al. (2003) affirment qu'il est possible de concilier la préservation et l'exploitation forestière dans certaines circonstances. Ainsi, Fredericksen et Puig (2001) proposent d'intensifier l'exploitation des forêts de production; ce qui favoriserait le renouvellement des populations d'espèces commerciales, permettrait de limiter l'exploitation à des zones contrôlées et contribuerait à mieux valoriser la forêt. Pour d'autres auteurs, au contraire, l'aménagement des forêts tropicales ne peut garantir en même temps la préservation de la biodiversité et la variabilité économique (Rice et al., 1998 ; Bawa et Seidler, 1998; Sist \& Brown, 2004 et Picard, 2007). Ils suggèrent une exploitation de type Cut and leave, c'est-à-dire une exploitation unique dans le temps, suivie d'une mise en réserve complète. La question principale est celle de l'évolution de la composition floristique des forêts après les perturbations. A l'échelle d'une communauté, c'est-à-dire d'un ensemble d'espèces en interaction dans un milieu donnée, l'ouverture du milieu provoque une augmentation de l'énergie lumineuse dans toutes les strates du couvert végétal. En conséquence, la composition spécifique peut alors être modifiée et déplacée vers un pôle plus héliophile (Molino et Sabatier, 2001).

Ainsi la gestion durable des forêts doit être fondée sur la connaissance de leur fonctionnement écologique. Les règles d'exploitations actuelles répondent principalement à une logique économique sans une réelle prise en compte ni des caractéristiques écologiques du milieu, ni des espèces. Elles consistent, Iorsqu'elles existent, à définir un diamètre minimum d'exploitation, une liste d'espèces à exploiter et éventuellement une durée de rotation, c'est-àdire une période de temps séparant deux passages d'exploitation.

De nombreux dispositifs de recherche et d'expérimentation ont été mis en place il y a environ quarante ans dans le but d'évaluer les effets des interventions sylvicoles dans plusieurs types de forêts en zone intertropicale (Picard, 2007). Les paramètres démographiques des peuplements suivis, obtenus en condition témoin et suite à des interventions sylvicoles, ont permis d'élaborer des modèles de dynamique forestière à Paracou en Guyane Française (Flores, 2005) et à Irobo et Mopri en Côte d'Ivoire (Durrieu de Madron et al., 1998 a et b).

Le choix du dispositif permanent de la Téné s'explique par la localisation de la zone d'étude, dans une forêt semi-décidue riche en espèces de valeur d'une part, et d'autre part la réponse émise par la perturbation créée par les traitements sylvicoles (1978) et le passage d'un feu de forêt en 1983.

L'objectif général de cette étude est d'évaluer l'impact de la perturbation par les traitements sylvicoles et le feu de forêt sur la dynamique du peuplement. Concrètement, l'étude tentera de répondre aux questions suivantes: (i) Peut-on mettre en évidence l'impact de l'intervention sylvicole sur la dynamique d'un peuplement forestier? (ii) Quels sont les impacts de ces deux types de perturbations sur la dynamique des essences? (iii) Enfin, le recul temporel dont nous disposons nous permet-il d'observer une restauration progressive des essences au sein des parcelles perturbées?

\section{Matériel et Méthodes}

\subsection{Présentation du site d'étude}

Le site de la présente étude se situe entre $5^{\circ} 17^{\prime} 32^{\prime \prime}$ 'et $5^{\circ} 29^{\prime} 21^{\prime \prime} \mathrm{W}$ et entre $6^{\circ} 30^{\prime} 51^{\prime \prime}$ 'et $6^{\circ} 31^{\prime} 56^{\prime \prime} \mathrm{N}$, dans la forêt classée de la Téné, au Centre-Ouest de la Côte d'Ivoire. La forêt classée de la Téné couvre une superficie de 29400 ha. 
Elle est caractéristique d'une forêt dense semidécidue (Anonyme 1995). Selon Guillaumet et Kahn (1979), le massif de la Téné fait le lien entre la forêt à Celtis spp. (Ulmaceae) et Triplochiton scleroxylon K.Schum. (Sterculiaceae) d'une part et la forêt à Nesogordonia papaverifera (A.Chev.) Capuron ex N.Hallé (Sterculiaceae) et Khaya ivorensis A.Chev. (Meliaceae) d'autre part.
Le dispositif repose sur un relief de bas plateaux granitiques. Les précipitations moyennes annuelles à la Téné varient entre 1200 et 1600 $\mathrm{mm}$. Elles sont réparties selon un rythme à quatre saisons dont deux saisons sèches (décembre à février et de juillet à aout) et deux saisons de pluies (mars à juin et septembre à novembre). La température moyenne annuelle est de $26^{\circ} \mathrm{C}$.

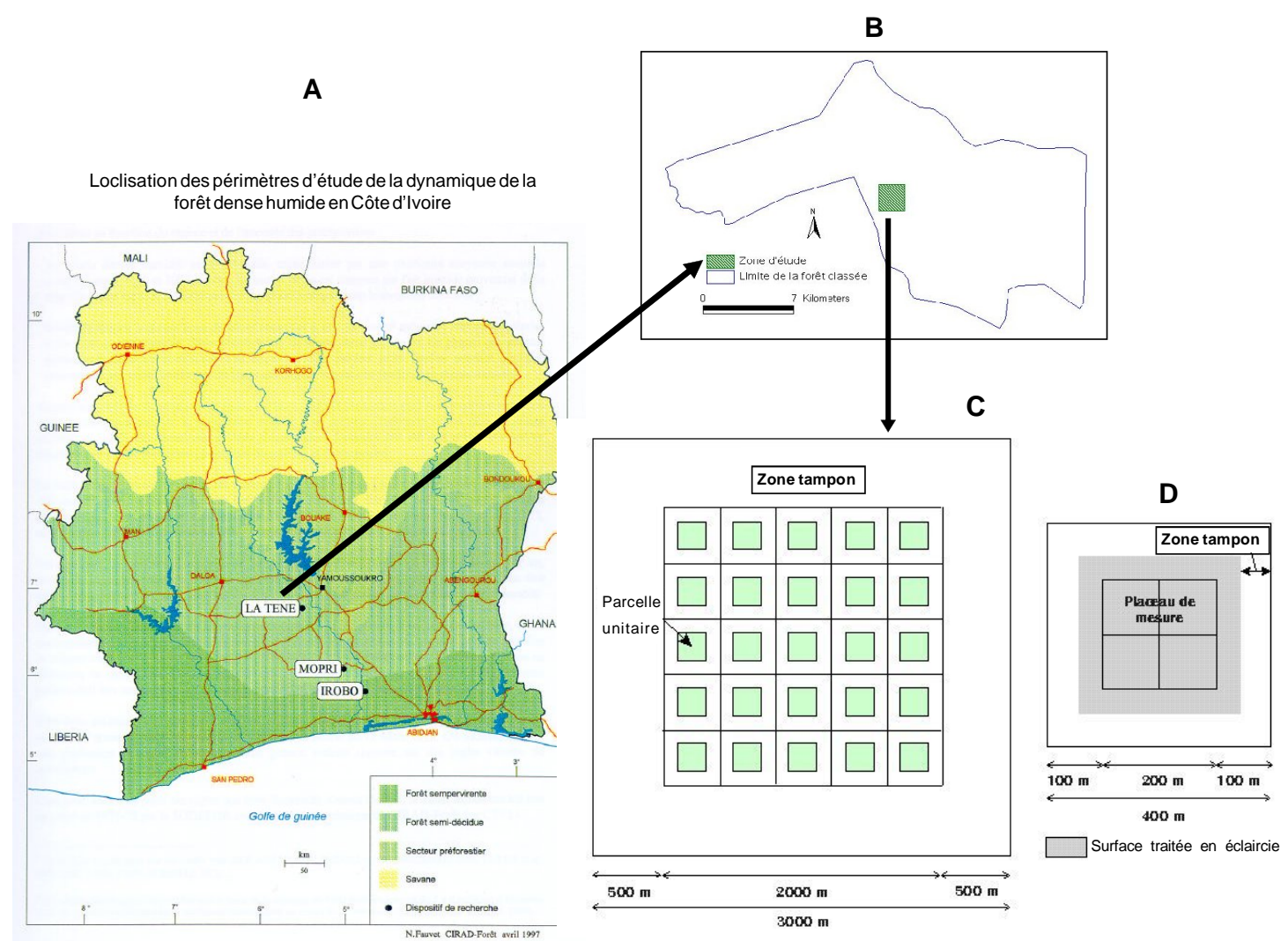

Figure1: Localisation et plan du dispositif permanent de Téné

A) Localisation des dispositifs permanents en Côte d'Ivoire. (B) Délimitation de la forêt classée de la Téné et localisation de la zone d'étude. (C) Plan du dispositif permanent de la Téné comportant vingt cinq parcelles reparties sur une superficie de 400 hectares. (D) Subdivision d'une parcelle de quatre hectares en quatre carrés d'un hectare et délimitée par une zone tampon.

\subsection{Le dispositif permanent}

Le dispositif permanent de la Téné fait partie d'un réseau de trois dispositifs (avec ceux de Mopri et Irobo) qui a été mis en place par la Société de développement des forêts (SODEFOR) en partenariat avec le Centre technique des forêts tropicales (CTFT) actuel Centre de coopération internationale en recherche agronomique et de développement (CIRAD). II couvre une superficie totale de 400 ha $(2 \mathrm{~km} \times 2 \mathrm{~km})$. II comporte une zone tampon de $500 \mathrm{~m}$ qui entoure 25 parcelles unitaires d'une surface de 16 ha chacune (Fig.1). Les mesures des diamètres ont été réalisées dans les 4 ha centraux de chaque parcelle unitaire (eux-mêmes subdivisés en sous-placeaux d'un hectare). Les traitements sylvicoles ont été en 
revanche appliqués sur la surface totale des parcelles unitaires (soit 16 ha) pour tenir compte des effets de bordure. Les essences présentes dans le dispositif ont été réparties en deux groupes en fonction de leurs caractéristiques technologiques:

- les essences commerciales (ou principales) sont celles commercialisées ou à promouvoir du fait de leurs caractéristiques technologiques intéressantes ;

- les essences secondaires sont celles qui ne sont pas actuellement commercialisées en bois d'œuvre.

\subsection{Traitements effectués}

La forêt de la Téné avait déjà fait l'objet d'une exploitation forestière de faible intensité peu de temps avant la mise en place du dispositif (Bertault et al., 1999). Lors de la mise en place du protocole d'étude en 1978, deux types d'interventions sylvicoles ont été réalisés:

- l'exploitation contrôlée d'essences commerciales a été effectuée dans dix parcelles ;

- l'éclaircie par dévitalisation sur pied des essences ont été faite dans cinq parcelles ;

- les dix parcelles restantes sont les parcelles témoins, conservées à titre de comparaison.

La surface terrière moyenne initiale du peuplement avant l'intervention sylvicole était de $28 \mathrm{~m}^{2} / \mathrm{ha}$. Onze campagnes de mesures, représentant une durée de vingt six ans étaient disponibles pour notre étude, en plus de celle effectuée avant intervention sylvicole par Mielot et Bertault (1980).

Dans ces parcelles exploitées, les essences commerciales de diamètre à hauteur de poitrine $(\mathrm{DBH})>80 \mathrm{~cm}$ ont été exploitées au taux de 12 à $41 \%$ de la surface terrière totale, soit un taux moyen d'extraction de $5,8 \mathrm{~m}^{2} / \mathrm{ha}$. L'exploitation a ramené la surface terrière des parcelles entre 17 et $25 \mathrm{~m}^{2} / \mathrm{ha}$. La surface terrière endommagée par l'exploitation était en moyenne de $2,1 \mathrm{~m}^{2} / \mathrm{ha}$, soit un total de $7,9 \mathrm{~m}^{2} /$ ha détruits ou exploités. Le volume moyen prélevé a été de $53 \mathrm{~m}^{3} /$ ha sur 160 ha (Bertault et al., 1999)
L'objectif des Parcelles éclaircies était de favoriser la croissance des essences commerciales en réduisant l'importance des essences secondaires. Dans ce dispositif, l'éclaircie a été réalisée par dévitalisation des arbres sur pied. Cette opération a été réalisée de manière systématique en commençant par les arbres de gros diamètre des essences secondaires jusqu'à l'obtention du pourcentage souhaité. Dans certains cas, en particulier pour les fortes éclaircies, une dévitalisation de certaines essences de moindre valeur commerciale a été nécessaire. L'éclaircie a ramené la surface terrière des parcelles à 15-21 $\mathrm{m}^{2} / \mathrm{ha}$, par la suppression de $8,6 \mathrm{~m}^{2} / \mathrm{ha}$ en moyenne soit environ $30 \%$ de la surface terrière initiale (Bertault et al., 1999).

Ainsi, les essences commerciales de plus de $\mathrm{DBH}>10 \mathrm{~cm}$ ont été identifiées et géopositionnées au mètre près ; leurs diamètres ont été mesurés à $50 \mathrm{~cm}$ au-dessus des contreforts.

Pour les essences secondaires de $\mathrm{DBH}>10 \mathrm{~cm}$ de diamètre, seule leur répartition en classe de diamètre a été effectuée.

Selon Bertault et al., 1999, après le passage du feu, les surfaces affectées par le feu de brousse en 1983 ont été estimées par parcelles en pourcentage ; cela a été utile pour l'analyse des données.

\subsection{Analyses des données}

Le diamètre est la seule grandeur mesurée sur les arbres. Les paramètres qui ont été calculés au niveau des parcelles sont les effectifs, la densité et la surface terrière.

Pour les essences commerciales, la surface terrière $\left(S T_{1}\right)$ exprimée en $\mathrm{m}^{2} /$ ha a été obtenue par la formule suivante :

$$
\mathrm{ST}_{1}=\sum_{i} g i=\sum_{i} \frac{p i d i^{2}}{4}
$$

avec $\frac{p i d i^{2}}{4}$, la surface terrière de chacun des arbres (gi) inventoriés, (di), le diamètre de chaque arbre et la constante $p i$ soit 3,14 . 
Pour les essences secondaires, le calcul des effectifs à la surface terrière $\left(\mathrm{ST}_{2}\right)$ a été effectué selon la méthode de calcul utilisée par Miélot et Bertault (1980) et Zobi (2002). Pour chaque classe de diamètre, ces auteurs ont utilisé la moyenne quadratique des limites inférieure $\left(d_{\min }\right)$ et supérieure $\left(\mathrm{d}_{\max }\right)$. La surface terrière totale de la classe de diamètre (i) s'obtient alors par le produit de la moyenne quadratique correspondante par l'effectif des arbres de cette classe $\left(n_{\mathrm{i}}\right)$ :

$$
\mathrm{ST}_{2}=\sum_{i} \frac{\operatorname{pini}\left(d_{\min }^{2}+d_{\max }^{2}\right)}{8}
$$

La surface terrière totale (STT) est la somme des surfaces terrières $S T_{1}$ et $S T_{2}$. Les variables $S T_{1}$ $\mathrm{ST}_{2}$ et STT ont été simultanément représentées par traitement (éclaircie, exploitation et témoin) afin de permettre les comparaisons et de mettre en évidence les fluctuations éventuelles au cours du temps.

Pour étudier l'effet de l'intensité du feu de forêt sur la dynamique du peuplement, les parcelles ont été subdivisées en deux groupes et une comparaison a été faite entre les parcelles affectées par le feu à plus de $45 \%$ à celles de moins $45 \%$. Des régressions linéaires simples et un test de corrélation ont permis de mettre en évidence l'existence d'une relation entre les groupes essences en fonction des années. Une analyse de variances (ANOVA) a servi à étudier les différences éventuelles entre la variable dépendante (surface terrière) et les variables explicatives (années, essences, traitements et effet du feu). La comparaison des moyennes a été faite avec la méthode de Bonferroni. De plus, la loi de distribution normale (test de Lilliefors non significatif $p>0,05$ ) et d'homoscédasicité (égalité des variances) ont été remplies afin d'appliquer ces analyses. Tous ces tests statistiques ont été effectués avec les logiciels Statistica 6.1 et Spss12.

\section{Résultats}

\subsection{Impact de l'intervention sylvicole sur le peuplement}

L'analyse de variance montre qu'il n'existait pas de différence significative $(p=0,42)$ entre les traitements avant l'intervention sylvicole, par contre en 1978 une différence significative $(p=0,01)$ entre les parcelles témoins et traitées. L'intervention sylvicole a modifié la structure initiale du peuplement par la réduction de moitié de la surface terrière au sein des parcelles traitées (Tableau 1).

Les parcelles exploitées et celles éclaircies présentent une différence significative $(p=0,001)$ Les parcelles éclaircies ont une surface terrière moins importante que les parcelles exploitées.

Tableau 1 : Comparaison de l'évolution de la surface terrière en fonction des traitements au cours du temps.

\begin{tabular}{lccccc}
\hline & 1977 & 1978 & 1986 & 1994 & 2004 \\
\hline Eclaircie & $43,86 \mathrm{a}$ & $17,37 \mathrm{a}$ & $9,66 \mathrm{a}$ & $16,95 \mathrm{a}$ & $20,18 \mathrm{a}$ \\
Exploitation & $41,65 \mathrm{a}$ & $25,35 \mathrm{~b}$ & $13,75 \mathrm{a}$ & $22,13 \mathrm{a}$ & $24,76 \mathrm{a}$ \\
Témoin & $43,86 \mathrm{a}$ & $35,51 \mathrm{~b}$ & $23,73 \mathrm{~b}$ & $30,94 \mathrm{~b}$ & $31,29 \mathrm{~b}$ \\
P-value & $>0,05$ & $<0,05^{*}$ & $<0,05^{*}$ & $<0,05^{*}$ & $>0,05^{*}$ \\
\hline
\end{tabular}

Le test effectué est une analyse de variance (ANOVA) entre traitements par date : 1977 ( $p>0,05), 1978(p<0,05), 1986$ ( $p<0,05), 1994$ $(p<0,05), 2004(p<0,05)$. Le signe $\left(^{*}\right)$ montre qu'il existe une différence significative. Les valeurs indiquées sont les surfaces terrières moyennes par année dans chaque traitement. Les lettres $(a, b)$ indiquent les résultats des tests de comparaison des moyennes.

\subsection{Impacts combinés du temps et des traitements sur la dynamique}

La représentation graphique de la surface terrière totale en fonction du temps (Fig.2) décrit une dimunition de la surface terrière après les interventions sylvicoles de 1978. Cette diminution se poursuit jusqu'en 1986 et une remontée en 1994. Le test d'ANOVA montre qu'il existe une différence significative $\left(p=1.10^{-4}\right)$ entre les surfaces terrières des différentes dates. La comparaison des moyennes de la surface terrière montre trois périodes bien distinctes : la première phase (1977-1978) est caractérisée par l'intervention sylvicole, la seconde (1986-1994) traduit le passage du feu et la dernière (19942004) explique la stabilité du milieu naturel.

En fonction des traitements, la dynamique du peuplement s'est différenciée de 1978 à 2004 (Fig.3). Le test d'ANOVA indique qu'il existe des différences significatives entre les surfaces terrières en fonction des traitements $\left(p=1.10^{-5}\right)$. Le test de comparaison des moyennes (Tableau 1) 
montre qu'il n'existe pas de différence entre les surfaces terrières avant l'intervention en 1977. Mais des différences significatives sont observées en 1978, 1986, 1994 et en 2004 entre les parcelles traitées et témoins. L'intervention sylvicole et le feu ont eu un impact sur les parcelles traitées et le milieu s'est équilibré au cours du temps après 1986; ce qui explique un fort recrutement dans ces parcelles.

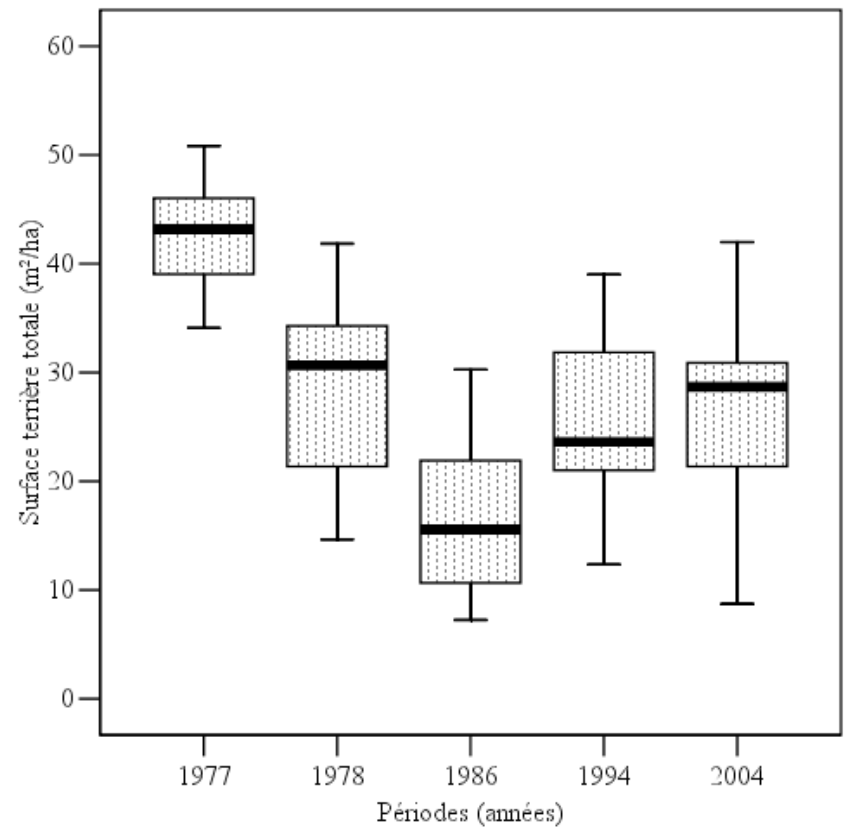

Figure 2 : Evolution de la surface terrière totale au cours du temps.

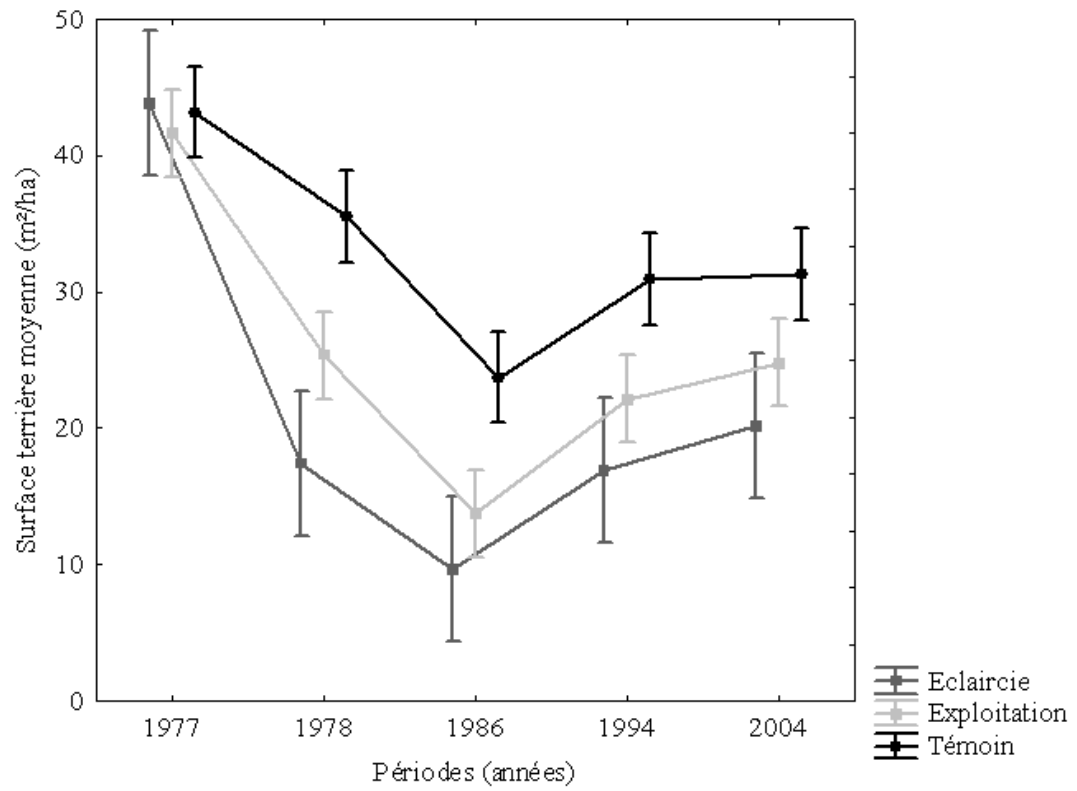

Figure 3 : Evolution de la surface terrière totale en fonction des traitements et dans le temps. 


\subsection{Impact de la perturbation sur la dynamique des essences}

La surface terrière des essences commerciales et secondaires en fonction des années (Fig.4) évolue suivant des allures différentes jusqu'en 1994 où la surface terrière des essences secondaires est plus importante que celle des essences commerciales. L'analyse de variances a permis de montrer des différences entre les années $\left(p=10^{-3}\right)$, les traitements $\left(p=10^{-5}\right)$ et les essences $\left(p=10^{-5}\right)$ et l'existence d'une interaction $(p=0,075)$ entre les années et les essences. La comparaison des moyennes montre qu'il existe des différences significatives $\left(p=1.10^{-5}\right)$ entre les essences en 1977, 1978 et en 1986. Par contre, il n'existe pas de différences $\left(p=2.10^{-5}\right)$ en 1994 et 2004. A court terme, les perturbations créées par l'intervention sylvicole (initialement) et plus tard par le feu ont été plus favorables aux essences commerciales mais à long terme (en 1994), cette tendance s'est inversée au profit des essences secondaires. Les résultats du test de Pearson montrent qu'il n'existe pas de corrélation $(r=-0,07$; $p=0,52)$ en 1977 et en 1978 ( $r=0,29 ; p=0,08)$ entre ces deux groupes d'essences ; mais une corrélation significative est observée entre eux en $1986(r=0,69$; $\left.p=3.10^{-3}\right), 1994\left(r=0,93 ; p=1.10^{-4}\right)$ et $2004(r=0,84$; $\left.\mathrm{p}=1.10^{-5}\right)$. L'intervention sylvicole a été défavorable aux essences secondaires mais le passage du feu a un impact sensible sur la composition floristique et surtout sur leur accroissement.

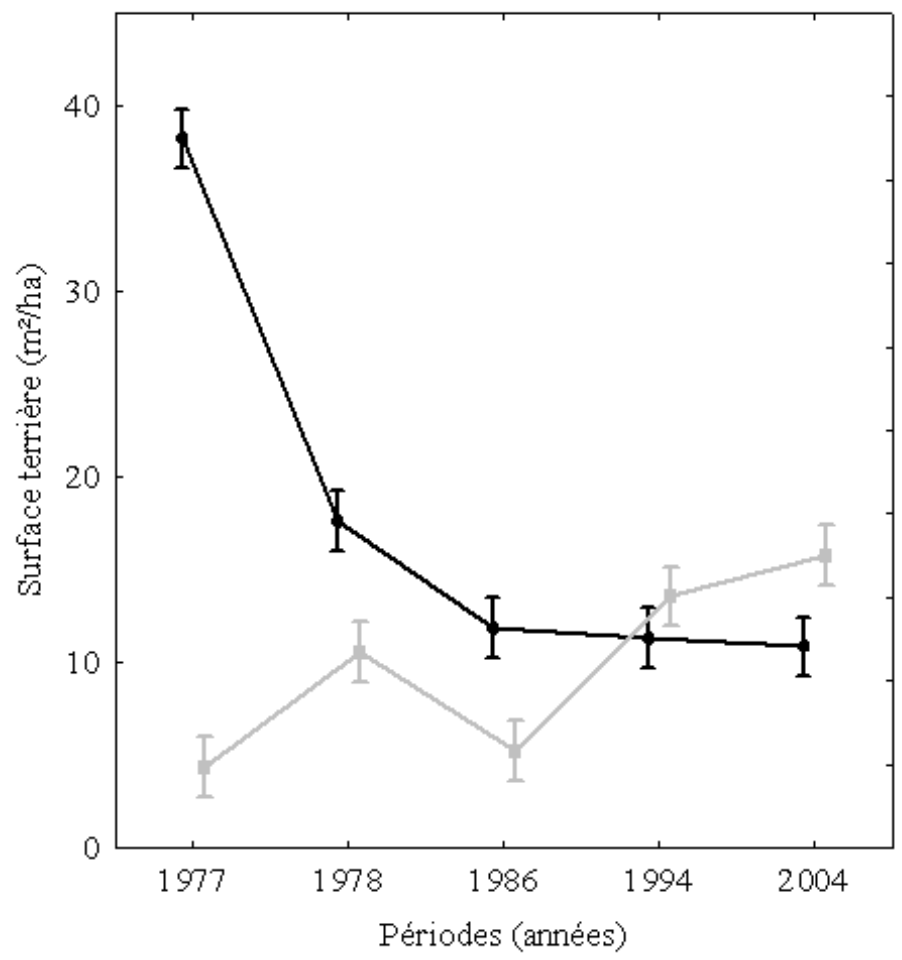

포 Essences commerciales
Essences secondaires

Figure 4 : Comparaison de la surface terrière des essences principales, secondaires en fonction des années.

\subsection{Impact du feu sur la dynamique du peuplement}

Les résultats obtenus montrent une différence significative $(p=0,01)$ entre l'effet du feu et en fonction des années mais il n'existe pas d'interaction $(p=0,55)$ entre l'effet du feu et les années. Le test de comparaison des moyennes montre une différence $(p=0,01)$ entre 1977 et les autres années. L'intervention sylvicole a modifiée la structure au sein des deux groupes de parcelles, le passage du feu a accentué cette modification. La surface terrière du peuplement de 1994 à 2004 tend vers celle observée après l'intervention sylvicole. 
La comparaison de l'état d'évolution de la surface terrière (Fig.5) entre les essences commerciales et secondaires présente des allures différentes. Au-delà de 1994, les essences secondaires présentent des surfaces terrières plus importantes au sein des parcelles affectées par le feu.

Le test d'ANOVA effectué entre les deux groupes d'essences, l'effet du feu et les années montre qu'il existe une différence significative $(p=0,02)$ entre les années, l'effet du feu et des essences alors qu'il n'y a pas d'interaction $(p=0,67)$ entre ces trois variables explicatives. Une différence significative $(p=0,001)$ est observée entre les deux groupes d'essences et entre les parcelles à forte et moindre intensités. Les parcelles qui ont été perturbées présentent une surface terrière plus importante avec les essences secondaires. Les nouvelles conditions du milieu naturel ont favorisées la croissance rapide de ces essences.

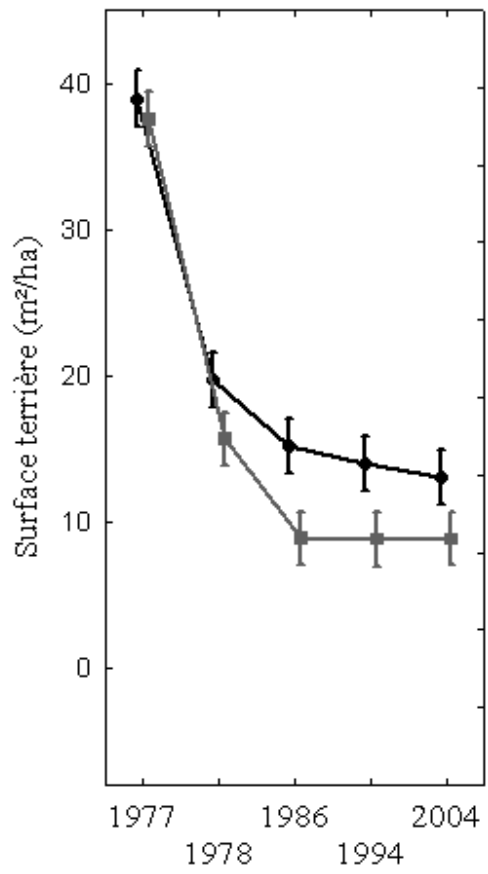

Essences commerciales

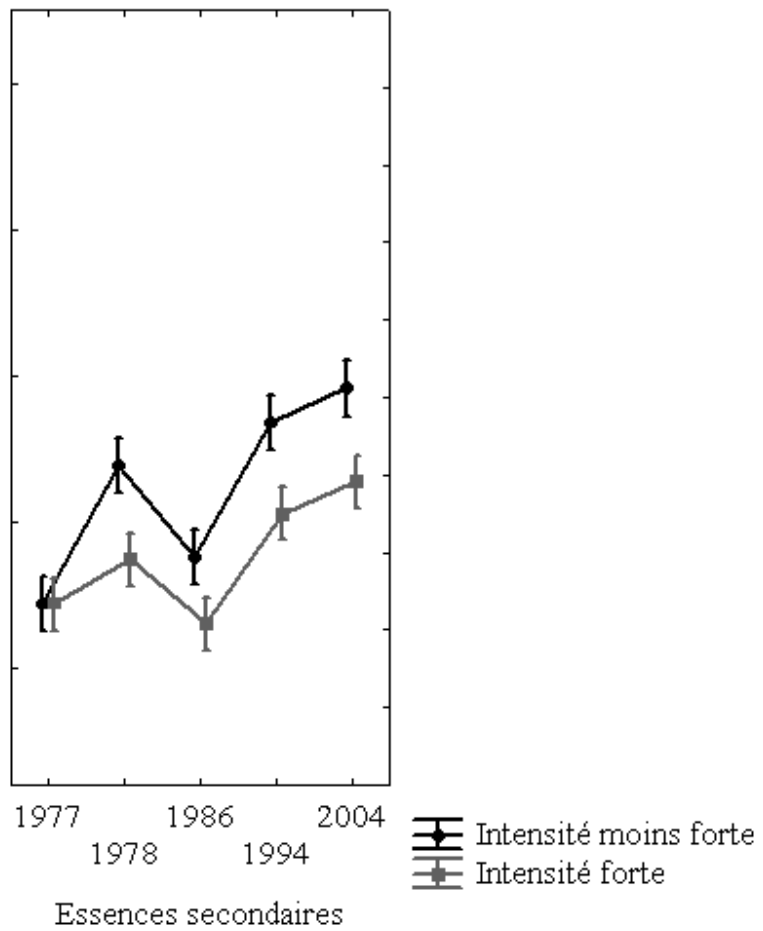

Essences secondaires

Figure 5 : Comparaison de l'évolution de la surface terrière des essences commerciales et secondaires en fonction de l'intensité du feu.

\section{Discussion}

Cette étude a permis de mettre en évidence, d'une part, l'impact de l'exploitation forestière sur la régénération naturelle des essences à vocation commerciale et secondaires et, d'autre part, d'identifier les dégâts causés par les traitements sylvicoles dans une forêt dense semidécidue de Côte d'Ivoire. L'intervention sylvicole dans le dispositif de la Téné a eu un impact négatif sur la dynamique du peuplement à cause de l'importante élimination des essences dans les parcelles éclaircies (20 à $30 \%$ ) soit 4 à 5 tiges/ ha et celles exploitées (10 à $20 \%$ ) soit 2 à 4 tiges/ha. L'évolution du peuplement au cours du temps a eu une influence sur l'accroissement des essences. Comparativement aux valeurs rencontrées ailleurs dans les forêts tropicales, Puig (2002) montre qu'en Afrique, le taux de prélèvement de 1 à 2 tiges/ha, soit environ 15$20 \mathrm{~m}^{3} / \mathrm{ha}$, est faible par rapport à celui pratiqué en Amérique où ce taux atteint 5 à 6 arbres/ha, 
soit environ $30-50 \mathrm{~m}^{3} /$ ha (Durrieu de Madron et al., 1998 ; Uhl et Vieira, 1989 ; Johns et al., 1996). L'une des contraintes majeures des prélèvements est l'hétérogénéité du peuplement forestier; les arbres n'y ont pas une distribution homogène comme dans les plantations. De plus, eu égard aux dégâts causés par les prélèvements et le faible taux de croissance des essences, le cycle de reconstitution de la végétation dans le cadre d'une gestion durable est long, généralement supérieur à 60 ans. L'exploitation forestière qui ne devrait pas empêcher la forêt de se régénérer cause toutefois des dégâts sur le potentiel de régénération (Puig, 2001).

L'intervention sylvicole provoque des ouvertures dont l'une des conséquences est la modification du microclimat forestier par un apport brusque de lumière. Il en résulte, notamment, une réactivation de la croissance des plantules, jeunes plants et autres rejets encore sur pied (potentiel végétatif). Les jeunes individus montrent la plus forte capacité de réaction à ces changements. La réactivation se poursuit jusqu'à la fermeture du couvert (Riera, 1995 ; Cruizat, 1966 ; Finkelstein, 1982 ; Whitmore, 1991).

L'exploitation forestière supprime une partie des strates supérieures (canopée et subcanopée) permettant à la lumière de pénétrer profondément dans le sous-bois, de lever la dormance de certaines graines au sol et d'accélérer leur germination. Le chablis en forêt naturelle procède de façon analogue mais sur des surfaces beaucoup moins importantes (Durrieu de Madron et al., 1998) en impliquant le potentiel séminal édaphique (les graines préexistantes dans le sol avant perturbation), le potentiel advectif représenté par les diaspores venues à la suite d'une perturbation et le potentiel végétatif correspondant aux plantules préexistantes avant perturbation qui se développent par la suite (Alexandre, 1982 ; Riera, 1983). Les plantules, les jeunes plants, les rejets et les arbres en place voient leur croissance stimulée de sorte que les ouvertures provoquées par l'intervention vont se refermer, parfois rapidement. Hall et Swaine (1981) observent dans trois types de forêts au Ghana (sempervirente, de transition et semi-décidue) que les germinations sont plus abondantes et les espèces plus nombreuses dans les ouvertures qu'en forêt fermée, quel que soit le type de forêt. Bertault (1999), Maître (1988, 1991 ) et Dupuy (1998) ont montré dans les dispositifs permanents d'Irobo et de Mopri, six et quatorze ans après l'intervention sylvicole, que les parcelles ayant connues une forte éclaircie ont montré un accroissement d'environ $15 \%$ du nombre de tiges de la régénération naturelle par rapport au témoin.

Au niveau de la composition floristique du peuplement, la régénération plus importante des essences commerciales pendant les dix premières années suivie par la prédominance des essences secondaires est contraire aux dispositifs de Mopri et d'Irobo où les essences secondaires occupent une place limitée entre 7 et $9 \%$ des effectifs (Bertault, 1999 ; Maître 1988, 1991 ; Dupuy 1998). Les éclaircies favorisent la régénération des espèces tolérantes à la lumière. L'effet de mise en lumière consécutif à l'intervention sylvicole favorise les espèces cicatricielles qui surmontent la concurrence des espèces pionnières grâce à leur vitesse d'installation et de croissance élevée. Dans les parcelles témoins, les semis survivants ont une croissance très faible et sont affectés par divers traumatismes (Puig et Fabre, 1996). Après l'intervention sylvicoles, les perturbations créées par l'ouverture de la voûte favorisent la germination d'espèces héliophiles à graines légères, souvent anémochores, telles que Terminalia superba, T. ivorensis (Combretaceae), Ceiba pentandra (Bombacaceae) au détriment d'autres essences commerciales telles que Entandrophragma spp et Khaya spp (Meliaceae).

Selon Siegert et al. (2001), les gigantesques incendies qui ont touché l'Indonésie en 1997 et 1998, ont démontré à large échelle que les zones forestières les plus affectées étaient celles qui ont été récemment exploitées. La perturbation du couvert a eu pour effet d'augmenter la quantité de combustible disponible (débris, recrus ligneux et herbacés, lianes) et d'augmenter la vitesse de dessèchement du combustible (Cochrane, 2003 ; Slik et Eichhorn, 2003 et Cochrane et Barber, 2009). Le résultat de ces effets combinés a augmenté la probabilité et l'impact des feux comparable au dispositif de la Téné où les parcelles traitées ont été plus vulnérables au feu à cause de l'abondante de la biomasse morte sur le sol (Bertault et al., 1999).

Les opérations sylvicoles telles que l'exploitation forestière peuvent aboutir à doubler la production et à favoriser le recrutement des espèces les plus intéressantes pour le sylviculteur (Maître, 
1991). Bariteau (1992) a observé que l'intervention sylvicole des parcelles pour toutes les espèces considérées induit une augmentation de la croissance relative des plantules d'autant plus importante que la sylviculture est intensive. Dans le dispositif permanent de la Téné, les traitements intensifs permettent, d'une part, le recrutement important des espèces cicatricielles et pionnières, et la diminution du taux d'essences sciaphiles préexistantes et stimulent, d'autre part, la croissance en hauteur des jeunes plants.

\section{Conclusion}

Cette étude avait pour objectif de déterminer l'effet de la perturbation occasionnée par les interventions sylvicoles et le passage du feu de forêt sur la dynamique du peuplement. Elle devait répondre à l'hypothèse selon laquelle les perturbations intervenues au sein de l'écosystème forestier occasionneraient des changements au niveau de la dynamique et de la composition floristique du peuplement au cours du temps. Les résultats ont montré que l'intervention sylvicole a eu un impact négatif sur la surface terrière au sein des parcelles éclaircies et exploitées. Le nombre d'arbres prélevés a sensiblement modifié la structure initiale des parcelles traitées. En 1978, les perturbations créées par l'intervention sylvicole et par le feu de forêt ont été plus favorables à la recrudescence des essences commerciales et au delà de 1994, au profit des essences secondaires. Ainsi, l'accroissement en volume a été plus important dans les milieux plus ouverts avec des essences à croissance rapide. Les nouvelles conditions naturelles du milieu ont permis aux essences secondaires d'occuper des espaces plus importants que les essences commerciales.

En somme, en tenant compte des paramètres de la dynamique du peuplement, il serait prématuré, après cette étude, d'affirmer que le dispositif s'est reconstitué par rapport à l'état initial. Vu l'ampleur créée par la perturbation, le dispositif permanent de la Téné mériterait d'être maintenu et les études sur d'autres aspects de la dynamique d'un peuplement forestier devraient être poursuivies afin d'apporter des éléments de réponses nouvelles pour les recherches futures.

\section{Remerciements}

Nous remercions la Société de Développement des Forêts (SODEFOR), l'Organisation Internationale des Bois Tropicaux (OIBT) et l'État de Côte d'Ivoire pour leur contribution à la réalisation de cette étude.

\section{Références citées}

Alexandre D. Y., 1982. Etude de l'éclairement du sous-bois d'une forêt dense humide sempervirente (Taï, Côte d'Ivoire). Acta Oecologica, vol.3, pp. 407- 447.

Anonyme 1995. Plan d'Aménagement de la forêt classée de la Téné (29.700 ha) 1994-2006. SODEFOR, Document interne, $43 \mathrm{p}$.

Bariteau M., 1992. Régénération de la forêt tropicale humide de Guyane. Etude des répartitions spatiales de trois espèces (Qualea rosea, Eperua falcata, Symphonia globulifera), Ann. Sci. For., 49, pp.359-382.

Bawa K. et Seidler R., 1998. Natural Forest Management and Conservation of Biodiversity in Tropical Forests. Conservation Biology, 12, pp.46-55.

Bertault, J.G, Miézan, K., Dupuy, B., Durrieu de Madron, L., Amsallem, Amsallem, I., 1999. Croissance et productivité en forêt dense humide après incendie. Le dispositif de la Téné-Côte d'Ivoire (1978 - 1993). Série Forafri-CIRAD, N²0, $67 \mathrm{p}$.

Cochrane, M.A. 2003. Fire science for rainforests. Nature; Vol.421, pp. 913-919.

Cochrane, M. A. et Barber, C. P., 2009. Climate change, human land use and future fires in the Amazon. Global Change Biology 15 (3), pp. 601-612.

Cruiziat P., 1966. Note sur le microclimat de la strate inférieure de la forêt équatoriale comparé à celui d'une clairière. Biologica Gabonica, Tome II, (4), pp. 361-402.

Dupuy B., 1998. Base pour une sylviculture en forêt dense tropicale humide africaine. Série Forafri, document 4, CIRAD-CIFOR, Coopération Française, 328 p.

Durrieu de Madron L., Favrichon, V., Dupuy, B., Bar-Hen, A. et Maître, H. F., 1998a. 
Croissance et productivité en forêt dense humide: bilan des expérimentations dans le dispositif d'Irobo. Côte d'lvoire (19781990). Série Forafri-CIRAD, $N^{\circ} 2,77 \mathrm{p}$.

Durrieu de Madron L., Favrichon, V., Dupuy, B, Bar-Hen, A. et Maître H. F., 1998b. Croissance et productivité en forêt dense humide : bilan des expérimentations dans le dispositif de Mopri. Côte d'Ivoire (1978-1990). Série Forafri-CIRAD, $\mathrm{N}^{\circ} 3,77 \mathrm{p}$.

Flores O., 2005. Déterminisme de la régénération chez quinze espèces d'arbres tropicaux en forêt guyanaise : les effets de l'environnement et de la limitation par la dispersion. Thèse à l'université Montpellier II, 264 p.

Finkelstein D., 1982. Evolution comparée des microclimats en coupe rase, sous recrû naturel et sous forêt primaire sur la parcelle Arbocel. Bulletin de Liaison Ecerex, $\mathrm{n}^{\circ} 6$, pp. 19-32.

Fredericksen T. et Putz F., 2003. Silvicultural intensification for tropical forest conservation. Biodiversity and conservation, 12, pp. 14451453.

Guillaumet J.L. et Kahn F., 1979. Description des végétations forestières tropicales. Approche morphologique et structurale. Candollea. 34(1) pp.109-131.

Hall J. B. et Swaine M. D., 1981 Distribution and ecology of vascular plants in a tropical rain forest. Forest vegetation in Ghana. La Haye, Ed. W. Junk, 383 p.

Hallé F., Oldeman R. A. A. and Tomlinson P.B., 1978. Tropical trees and Forest. An Architectural Analysis. Berlin, Springer Verlag, $441 \mathrm{p}$.

Johns J. F., Barreto P. and Uhl C., 1996. Logging damage during planned and unplanned logging operations in the eastern Amazon. For. Ecol.Manag., 89, pp. 59-77.

Maître H.F., 1988. Dynamique et Production des peuplements naturels de forêt dense humide en Afrique. Bois et Forêts des Tropiques, 213, pp. 3-12.

Maître H.F., 1991. Sylvicultural interventions and their effect on forest dynamics and production in some rain forest of Côte d'Ivoire In: GomezPompa A., Whitmore T.C. et Hadley M.(eds),
Rain Forest regeneration. Paris, MABUNESCO, pp. 383-393.

Mielot J. et Bertault J.G., 1980. Etude dynamique en vue de l'aménagement de la forêt dense de Côte d'Ivoire. But et description des travaux réalisés. SODEFOR, Côte d'Ivoire, 165 p.

Molino J.F. et Sabatier D., 2001. Tree diversity in tropical rain forest: A validation of the intermediate disturbance hypothesis. Sciences, pp.1702-1704.

Pearce D., Putz F. et Vanclay J., 2003.Sustainable forestry in the tropics: panacea or folly. Forest Ecology and Management, 172, pp. 229-247.

Picard, N., 2007. Dispositifs permanents pour le suivi des forêts en Afrique Centrale : un état des lieux. Rapport pour la COMIFAC. CIRAD, France, $38 \mathrm{p}$.

Poore D., Burgess P., Palmer J., Rietbergen S. et Synnott T., 1989. No timber without trees, Earthscan Publications Ltd, London, pp.1-10.

Puig H., 2001. La forêt tropicale humide, Belin, Paris, $440 \mathrm{p}$.

Puig H. et Fabre A., 1996. Survival and growth of seedling and sampling of Iryanthera hostmanii: A regeneration Strategy in French Guyana Rain Tropical Forest. Journal of Tropical Ecology, 13, pp.139-143.

Rice R., Sugal C. et Bowles I., 1998. Sustainable Forest Management: a Review of the Current Conventional Wisdom. Rapport technique, Conservation International, pp. 20-35.

Riera B., 1983. Chablis et cicatrisation en forêt guyanaise (piste de Saint-Elie). Thèse de doctorat de l'Université Paul Sabatier, Toulouse, $191 \mathrm{p}$

Riera B., 1995. Rôle des perturbations actuelles et passées dans la dynamique et la mosaïque forestière. Revue Ecologie, Terre et Vie, 50, pp. 209-222.

Siegert, F., Ruecker, G., Hinrichs, A. and Hoffmannk, A. A. 2001. Increased damage from fires in logged forests during droughts caused by el Niño. Nature Vol. 414, pp. 437- 440.

Sist P. et Brown N., 2004. Silvicultural intensification for tropical Forest conservation: a response to Fredericken and Putz. Biodiversity and conservation, 13, pp. 2381-2385. 
Slik, J. W. F. et Eichhorn, K. A. O., 2003. Fire survival of lowland tropical rain forest trees in relation to stem diameter and topographic position. Oecologia, 137, pp.446-455.

Uhl C. et Vieira I.G., 1989. Ecological impact of selective logging in Brazilian Amazon: a case study from the Paragominas region of the state of Para. Biotropica, 21, pp.98-106.

Whitmore T. C., 1991. Tropical rain forest dynamics and its implication for management In: GomezPompa A., Whitmore T.C. et Hadley M. (eds), Rain Forest regeneration. Paris, MAB-UNESCO, pp.67-89.

Zobi I.C., 2002. Contribution à la gestion sylvicole des forêts humides ivoiriennes à travers l'analyse des dispositifs permanents de Mopri et Irobo. Thèse de l'Université Claude Bernard- Lyon 1, France, $159 \mathrm{p}$. 